\title{
The Chemiluminescence Response of Human Platelets
}

\author{
Elaine L. Mills, Jonathan M. Gerrard, Dana Filipovich, James D. \\ White, and PaUl G. QuIE, Department of Pediatrics, University of Minnesota \\ School of Medicine, Minneapolis, Minnesota 55455
}

\begin{abstract}
A B S T RA C T Human platelets and platelet particulate fractions were found to emit a burst of chemiluminescence during incubation with arachidonic acid. The magnitude of light emission was directly related to the number of platelets in the reaction mixture and varied little for the same individual from day to day. The chemiluminescence response of platelets was localized to the particulate fraction and was almost totally oxygen dependent. In addition to arachidonate, seven other polyunsaturated fatty acids, including several that are not prostaglandin precursors, also induced platelet chemiluminescence.
\end{abstract}

A correlation was sought between chemiluminescence and platelet prostaglandin synthesis. Platelets incubated in low concentrations of aspirin, or platelets from subjects who had ingested aspirin, had markedly decreased arachidonic acid-induced chemiluminescence. Salicylic and sulfosalicylic acid had no inhibitory effect. A time-response curve of aspirin inhibition of arachidonate-induced chemiluminescence closely paralleled a time-response curve of aspirin inhibition of malondialdehyde production. Linoleic acid-induced platelet chemiluminescence was also markedly inhibited using aspirin-incubated platelets or platelets from subjects who had ingested aspirin.

These studies implicate activation of the enzyme prostaglandin synthetase in the arachidonate-induced platelet chemiluminescence. They provide evidence that linoleic acid may also specifically activate platelet cyclooxygenase to produce electronically excited species capable of light emission.

\section{INTRODUCTION}

Chemiluminescence $(C L)^{1}$ has been reported to accompany a number of normal enzymatic reactions in

Dr. Mills and Dr. Gerrard are recipients of Minnesota Heart Association Research Fellowship Awards.

Received for publication 25 April 1977 and in revised form 6 October 1977.

${ }^{1}$ Abbreviations used in this paper: CL, chemiluminescence; C-PRP, citrate-platelet-rich plasma; HBSS, Hanks' balanced salt solution; PMN, polymorphonuclear neutrophils. biologic systems including lipid peroxidation of liver microsomes (1-3) and adrenal mitochondria (4) as well as phagocytosis in human neutrophils $(5,6)$ and monocytes $(7,8)$. The photon emission of these CL reactions is thought to occur as a result of the decay of electronically excited species such as singlet oxygen or excited carbonyls. Thus, light production serves as an indicator of the presence of highly reactive, short-lived intermediate products present in these systems.

Recently, CL has been observed during the incubation of arachidonic acid with sheep vesicular gland microsomes (9), a system known to support prostaglandin biosynthesis. Light emission was blocked by singlet oxygen scavengers and by prostaglandin synthetase inhibitors and was felt to be related to an early event in prostaglandin biosynthesis. We now report production of CL by the addition of arachidonic acid to human platelets and the relationship of light emission to platelet prostaglandin synthesis.

\section{METHODS}

Platelet preparation. To prepare washed platelets or platelet-rich plasma, blood was obtained from normal donors who had not taken aspirin for 14 days. The blood was mixed immediately with dextrose-citrate buffer, $\mathrm{pH} 6.5$, in a ratio of nine parts blood to one part anticoagulant. Citrate-plateletrich plasma (C-PRP) was separated by centrifugation at $100 \mathrm{~g}$ for $20 \mathrm{~min}$ at $22^{\circ} \mathrm{C}$. Washed platelets were prepared by adding $1 \mathrm{ml} \mathrm{10 \%}$ EDTA (0.343 M, pH 7.4) to $9 \mathrm{ml} \mathrm{C-PRP,} \mathrm{centrifuging}$ at $1,110 \mathrm{~g}$ in a Sorvall RC-2 (Ivan Sorvall, Inc., Norwalk, Conn.) for $10 \mathrm{~min}$ at $4^{\circ} \mathrm{C}$, and resuspending the platelets in Hanks' balanced salt solution (HBSS; $\mathrm{pH} 7.4$ ) to make a final volume of $10-\mathrm{ml}$ separated platelets per $100 \mathrm{ml}$ whole blood drawn. Final platelet count, monitored by Coulter counting (model B, Coulter Electronics Inc., Hialeah, Fla.), was 1.6-2.0 × 10 platelets per vial. Examination of final platelet preparations using a Wright stain showed that the total neutrophil contamination was $0.05 \%\left(1 \times 10^{5}\right.$ polymorphonuclear neutrophils [PMN] per vial). Washed platelets and C-PRP were maintained at $4^{\circ} \mathrm{C}$ until $5 \mathrm{~min}$ before use when they were allowed to warm at room temperature. Platelets were used within 2-3 h after blood collection.

Particulate fraction of platelets. Platelet particulate fractions were prepared as described previously (10) except that they were resuspended in a $0.1 \mathrm{M}$ tris buffer $(\mathrm{pH} 8.5)$ without phenol.

Leukocyte preparation. Venous blood drawn from healthy 
volunteers was heparinized (10 $\mathrm{U}$ heparin $/ \mathrm{ml}$ blood), settled on $6 \%$ dextran $\left(0.3 \mathrm{ml} / 10 \mathrm{~cm}^{3}\right)$, centrifuged, and the leukocyte pellet washed once with $10 \mathrm{ml} 0.87 \% \mathrm{NH}_{4} \mathrm{Cl}$ to remove erythrocytes. The cells were resuspended in HBSS, counted, and adjusted to $1 \times 10^{7} \mathrm{PMN} / \mathrm{ml}$. For studies to control for leukocyte contamination in platelet preparations, leukocytes were prepared as above using one of two methods of centrifugation. In protocol $\mathrm{I}$, the leukocytes were centrifuged at $1,100 \mathrm{~g}$ for 10 min as in platelet preparation. In protocol II, leukocytes were centrifuged at $100 \mathrm{~g}$ for $10 \mathrm{~min}$ as for particle-induced neutrophil CL studies previously reported, (7) and used here to study neutrophils of a chronic granulomatous disease patient.

Preparation of fatty acids. All fatty acids (>99\% pure $\mathrm{NU}$ CHECK PREP, Elysian, Minn. and Sigma Chemical Co., St. Louis, Mo.) were made up as solutions of the sodium salt in tris buffer (0.015 M, pH 8.5) by the addition of an equimolar concentration of $1 \mathrm{M} \mathrm{NaOH}$ to the fatty acid. The final concentration of all fatty acids was $0.16 \mathrm{mmol} / \mathrm{ml}$. Sodium salts of unsaturated fatty acids were protected from exposure to air and light to minimize auto-oxidation, stored at $-70^{\circ} \mathrm{C}$ in small aliquots, and fresh aliquots used for each experiment.

CL assay. Fatty acids were added to dark-adapted glass scintillation vials that contained the washed platelets or platelet particulate fraction and enough phosphate-buffered saline (pH 7.4) to make a final volume of $5.5 \mathrm{ml}$ and a final $\mathrm{pH}$ of 8.0 . CL was measured in a $\beta$-counting spectrometer (model LS- $100 \mathrm{C}$, Beckman Instruments, Inc., Fullerton, Calif.) at ambient temperature $\left(22^{\circ} \mathrm{C}\right)$ with the counter adjusted out of coincidence, as described by Stanley and Williams (11) and Stjernholm et al. (12). Glass counting vials (161698, Beckman Instruments, Inc.) were dark-adapted for $24 \mathrm{~h}$ and filled under red illumination. Counting was done with an adjustable window opened wide to count the entire measurable light spectrum. Background readings were taken for the vials, additions were made, and the vials hand-agitated vigorously for $2 \mathrm{~s}$ to $\mathrm{mix}$ the platelet suspensions. Readings were taken at $0.1 \mathrm{~min}$ continuously for several minutes. Results were plotted as cpm $\times 10^{-3} \mathrm{vs}$. time. CL was expressed as peak activity throughout these studies rather than as an integral value. However, all results were entirely comparable when calculated by either method.

Preparation of additives. Aspirin was prepared immediately before use as a $10-\mathrm{mM}$ solution in $\mathrm{HBSS}$ at $\mathrm{pH} 7.4$. Salicylic acid and sulfosalicylic acid were prepared immediately before use as $10-\mathrm{mM}$ solutions in phosphate-buffered saline $\mathrm{pH} 7.4$.

Malondialdehyde formation. Malondialdehyde formation was studied using a modification of the method of Smith et al. (13). After addition of the arachidonic acid to $5.5-\mathrm{ml}$ washed platelets $\left(2 \times 10^{9}\right.$ platelets) and incubation for $5 \mathrm{~min}$, during which time CL was measured, $0.25 \mathrm{~g}$ bovine albumin was added. The suspension was mixed with an equal volume of $20 \% \mathrm{wt} / \mathrm{vol}$ trichloroacetic acid in $0.6 \mathrm{M} \mathrm{HCl}$, centrifuged, and the supernate assayed for malondialdehyde using the thiobarbituric acid reaction. Controls, including platelets alone and arachidonic acid plus buffer, were run with each reaction, and the malondialdehyde production assessed by subtracting the control values, and correcting for recovery.

\section{RESULTS}

\section{CL of platelets and platelet particulate fraction by arachidonic acid}

The addition of arachidonic acid to suspensions of human platelets produced a burst of CL. The mean peak CL response of 35 adult volunteers is shown in Fig. 1 . The magnitude of light production varied widely among the different donors but the same individuals varied little from day to day, and peak CL consistently occurred between 6 and $30 \mathrm{~s}$ after insertion of the vial in the counter (12-36 s after the addition of arachidonic acid). The magnitude of the CL response for any particular donor was directly related to the number of platelets in the reaction mixture when the concentration of arachidonic acid was held constant (Fig. 2).

The CL response of the washed platelets of a known chronic granulomatous disease patient was $130 \times 10^{-3}$ cpm, a value entirely comparable to normal platelet donors. This finding would correlate with the observed normal phagocitosis-stimulated nitroblue tetrazolium reduction by platelets from these patients (14). The neutrophils from this patient produced no measurable CL during phagocytosis of zymosan particles, an observation Stjernholm has also reported (12).

In an attempt to localize the platelet CL response to a particular subcellular fraction, separated platelets and a platelet particulate fraction were prepared from equal volumes of homologous blood. As illustrated in Fig. 3, a significant proportion of the luminescence was localized to the particulate fraction.

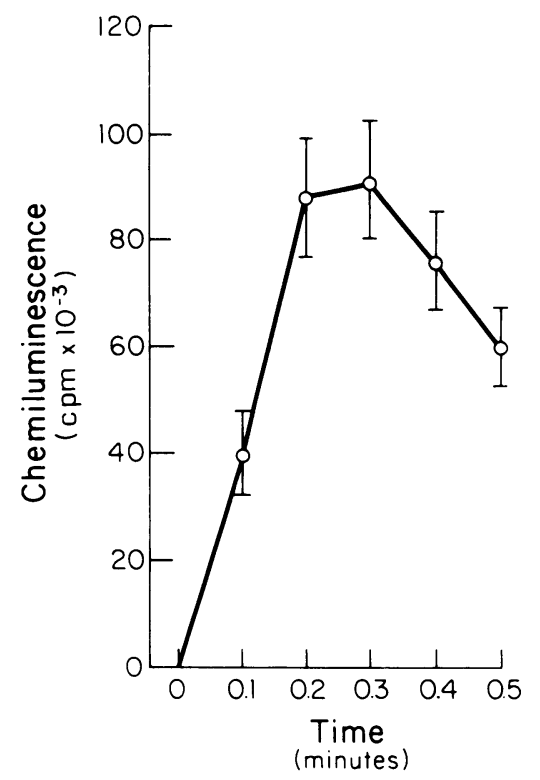

FIGURE 1 CL production of washed platelets induced by arachidonic acid. To each vial was added $\cong 2 \times 10^{9}$ washed platelets suspended in HBSS (pH 7.4) and enough phosphate-buffered saline $(\mathrm{pH} 7.4)$ to make a final volume of 5.5 $\mathrm{ml}$. The reaction was initiated by the addition of arachidonic acid as its sodium salt in a final concentration of 8 $\mu \mathrm{mol} / \mathrm{vial}$. The mixture was vigorously hand-agitated for $2 \mathrm{~s}$ before placing in a $\beta$-counting spectrometer at 0 time. Incubation was done at $22^{\circ} \mathrm{C}$. Each data point represents the mean value and $\mathrm{SE}$ of 35 experiments ( 35 donors). 


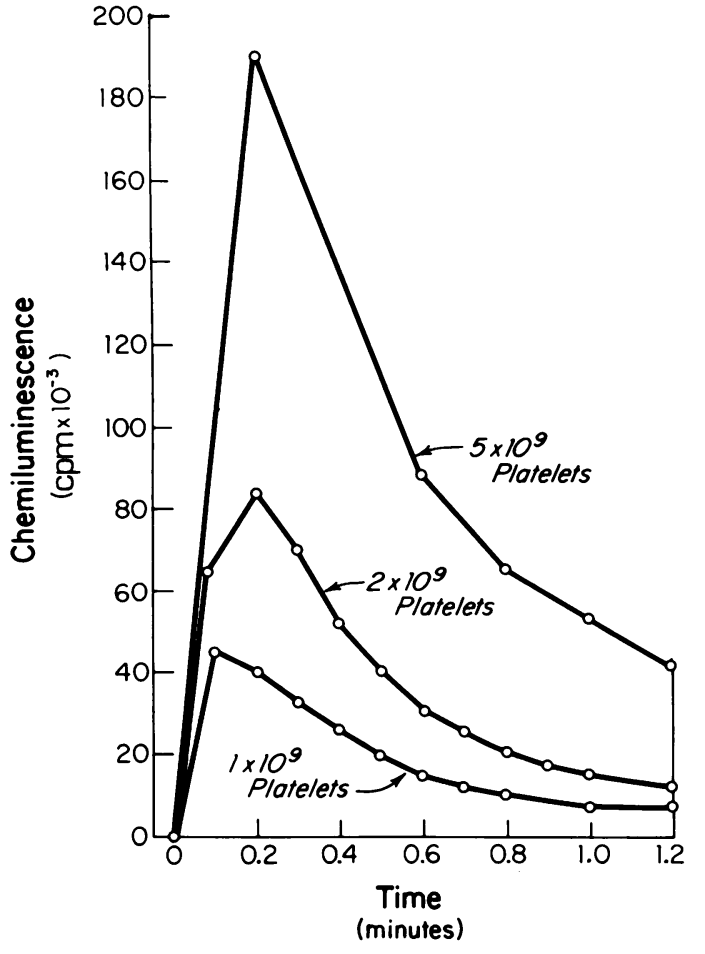

FIGURE 2 Dose-response curve of CL production induced by arachidonic acid using platelets from a single donor. The reaction mixtures were prepared exactly as in Fig. 1. Arachidonic acid was held at $8 \mu \mathrm{mol} / \mathrm{vial}$.

\section{Induction of CL in platelets by otherfatty acids}

In addition to arachidonic acid, eight other polyunsaturated fatty acids were studied in the same molar concentrations as arachidonic acid, and all were found to induce CL in platelets (Table I). Four saturated and two monounsaturated fatty acids did not induce CL when studied in concentrations of $8 \mu \mathrm{mol} / \mathrm{vial}$.

\section{Properties of platelet CL reaction systems}

Because not only the prostaglandin precursors, arachidonate, homo- $\gamma$-linolenate and adrenic acid (15, 16), but also a number of other polyunsaturated fatty acids were found to induce platelet $\mathrm{CL}$, it was necessary to explore the possibility that CL was a nonspecific reaction, initiated by a nonenzymatic substance in platelets during platelet-fatty acid incubation. When separated platelets suspended in HBSS were placed in a boiling water bath $\left(100^{\circ} \mathrm{C}\right)$ for $5 \mathrm{~min}$ before the addition of arachidonate, CL was reduced to $13 \%$ of control (Table II). These results suggest that almost $90 \%$ of the CL induced in platelets by arachidonate (and possibly by other polyunsaturated fatty acids) could be enzyme dependent.

Polyunsaturated fatty acids are extremely susceptible to auto-oxidation and could generate light-produc-

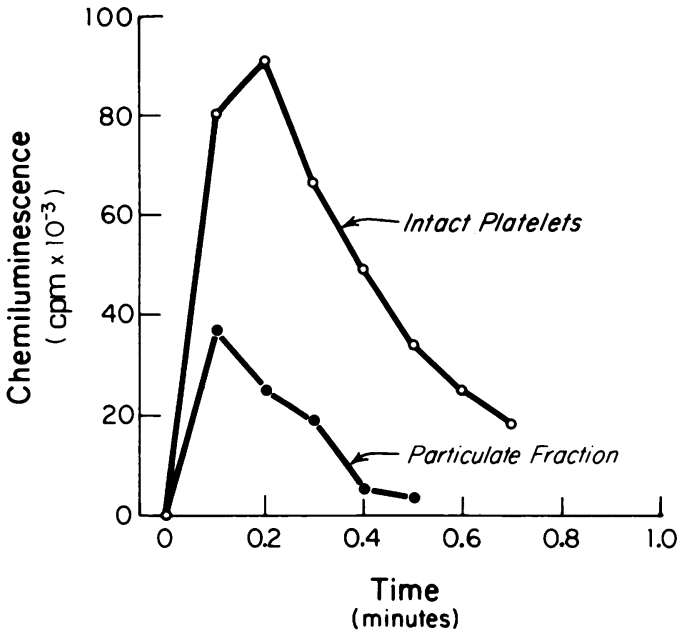

Figure 3 Comparison of the CL production of washed platelets with that of homologous platelet particulate fraction in the presence of arachidonic acid. The reaction mixtures were prepared and the conditions were maintained exactly as in Fig. 1. To each vial was added $2 \times 10^{9}$ platelets or $0.4 \mathrm{ml}$ platelet particles prepared from equal volumes of blood from the same donor.

ing species under certain experimental conditions. Inasmuch as arachidonic acid alone produced virtually no measurable CL (Table II), the possibility that spontaneous generation of CL from fatty acid oxidation was contributing to platelet CL was eliminated. However, the possibility that fatty acid auto-oxidation gave rise

TABLE I

The Effect of Various Fatty Acids on CL Production by Washed Platelets

\begin{tabular}{llcr}
\hline \multicolumn{1}{c}{ Fatty acid* } & Formula & CL peak & $\begin{array}{c}\text { Number of } \\
\text { experiments }\end{array}$ \\
\hline & & $c p m \times 10^{-3}$ & \\
Docosahexenoic & $22: 6 \mathrm{w} 3$ & 17 & 4 \\
Adrenic & $22: 4 \mathrm{w} 6$ & 54 & 2 \\
Arachidonic & $20: 4 \mathrm{w} 6$ & $91 \pm 11$ & 35 \\
Homo- $\gamma$-linolenic & $20: 3 \mathrm{w} 6$ & 66 & 3 \\
Eicosatraenoic & $20: 3 \mathrm{w} 3$ & 39 & 4 \\
Eicosadienoic & $20: 2 \mathrm{w} 6$ & 62 & 2 \\
$\gamma$-Linolenic & $18: 3 \mathrm{w} 6$ & 55 & 2 \\
Linolenic & $18: 3 \mathrm{w} 3$ & 59 & 2 \\
Linoleic & $18: 2 \mathrm{w} 6$ & $133 \pm 21$ & 13 \\
& & 3 & 2 \\
Gonodic & $20: 1 \mathrm{w} 9$ & 3 & 2 \\
Oleic & $18: 1 \mathrm{w} 9$ & 4 & 2 \\
Arachidic & $20: 0$ & 5 & 2 \\
Stearic & $18: 0$ & 3 & 2 \\
Palmitic & $16: 00$ & 4 & 2 \\
Myristic & $14: 00$ & 4 & \\
\hline
\end{tabular}

* All fatty acids were prepared as sodium salts to a final concentration of $8 \mu \mathrm{mol} / \mathrm{vial}$. 
TABLE II

Arachidonic Acid-Induced Platelet $C L$

\begin{tabular}{lc}
\hline \multicolumn{1}{c}{ Conditions } & CL \\
& $c p m \times 10^{-3}$ \\
Complete mixture* & 220 \\
Omit platelets & 6 \\
Omit arachidonic acid & 0 \\
Boiled platelets $\$$ & 28 \\
\hline
\end{tabular}

* The reaction mixture was prepared exactly as in Fig. 1 . $\$$ Washed platelets were placed in boiling water $\left(100^{\circ} \mathrm{C}\right)$ for $5 \mathrm{~min}$.

to hydroperoxides, which could generate CL reactions catalyzed by platelet enzymes or nonenzymatic substances in platelets, also had to be eliminated. Precautions were taken to prevent this from occurring by using only very purified preparations of fatty acids, only fresh or fresh-frozen preparations protected from exposure to air and light (Methods), and by using several different lots of arachidonic acid purchased from two different chemical companies. Although these precautions do not rule out the possibility of products of fatty acid oxidation initiating platelet $C L$, it makes this possibility unlikely.

\section{Presence of leukocytes in platelet suspensions}

Platelet preparations contained $<0.05 \%$ neutrophils $\left(1 \times 10^{5} \mathrm{PMN} / 2 \times 10^{9}\right.$ platelets per vial $)$. To estimate the possible contribution of neutrophil CL to the total platelet CL, neutrophils separated by centrifugation methods described in protocol I (Methods), as in platelet preparation, were incubated with arachidonic acid alone or added to standard platelet preparations. When $5 \times 10^{5}$ of these PMN were added to standard platelet-arachidonic acid mixtures, no increase was noted in peak CL. When arachidonic acid was added to $5 \times 10^{5}$ of these neutrophils suspended in HBSS in the absence of platelets, there was no measurable CL. Further, increasing the platelet leukocyte contamination by a factor of 10 by adding $5 \times 10^{6} \mathrm{PMN}$ to standard plateletarachidonic acid mixtures resulted in no measurable increase in CL. This data would suggest that virtually none of the measurable arachidonic acid-induced CL was the result of granulocyte luminescence.

The complete absence of detectable neutrophil CL in our platelet preparations may be due in part to neutrophil damage sustained by the more vigorous centrifugation used in the platelet preparation procedure. When neutrophils were prepared for particle-induced neutrophil CL (protocol II, Methods), there was no measurable increase in CL upon addition of $5 \times 10^{5}$ of these PMN to platelet preparations. However, there was a $10 \%$ increase in platelet CL when platelet-leuko- cyte contamination was increased 10 -fold by adding $5 \times 10^{6}$ of these PMN to the reaction mixtures.

The lack of detectable neutrophil CL in our platelet preparation is also largely due to the fact that neutrophils in this study are exposed to polyunsaturated fatty acids rather than target particles that induce phagocytosis (17). The addition of opsonized zymosan particles $(5 \mathrm{mg})$ to $5 \times 10^{5}$ neutrophils prepared according to protocol II, and measuring CL as previously described (7) for phagocytosis, induced small but detectable CL $\left(20 \mathrm{cpm} \times 10^{-3}\right)$. The addition of arachidonic acid to 5 $\times 10^{5}$ neutrophils prepared by protocol II again produced small but detectable CL. Thus, both the method of neutrophil preparation and the type of agent used to induce CL are critical to neutrophil light production.

\section{Oxygen requirement of $C L$}

The oxygen dependency of the platelet CL response was demonstrated by replacing the oxygen in the reaction system with gaseous nitrogen (Fig. 4). All reaction mixtures were prepared from the same platelet prepara-

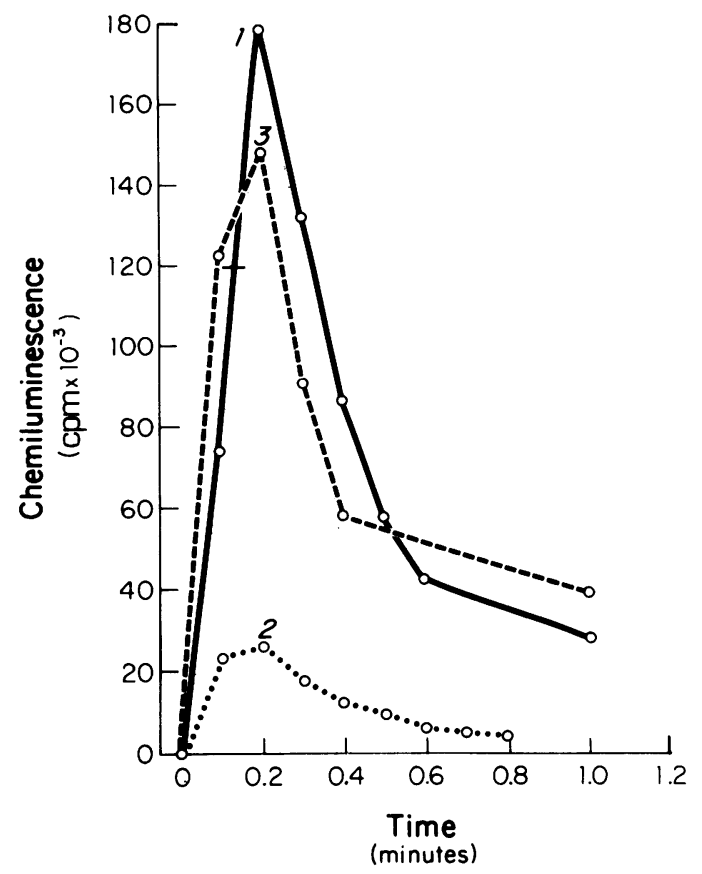

Figure 4 The influence of oxygen on CL. The reaction mixtures were all obtained from the same platelet preparation. The reaction of curve 1 was prepared and maintained under exactly the same conditions as in Fig. 1. Curve 2 was obtained by degassing the buffer $(20 \mathrm{~min})$, adding the platelets $\left(2 \times 10^{9}\right)$ to the buffer, and bubbling the mixture with nitrogen $(20 \mathrm{~min})$ before the addition of arachidonic acid at 0 time. Curve 3 was obtained exactly as curve 2 except after bubbling the mixture with nitrogen $(20 \mathrm{~min})$, the platelets were allowed to stand at atmospheric conditions (room air for $30 \mathrm{~min}$ ) before the addition of arachidonic acid at 0 time. 
tion. The reaction mixture, in which oxygen was largely replaced by gaseous nitrogen (Fig. 4 , curve 2 ), was obtained by degassing the buffer with nitrogen for $20 \mathrm{~min}$, adding the platelets to the buffer, and degassing that mixture as well. During the addition of arachidonic acid at 0 time, the reaction mixture was briefly exposed to atmospheric conditions. Thus, the system was not maintained entirely oxygen-free, and this may account for the small amount of CL produced. To demonstrate that the platelets were not damaged or inactivated by the nitrogen, an oxygen-depleted reaction mixture prepared as above was allowed to equilibrate with room air for $30 \mathrm{~min}$. When arachidonic acid was added to these platelets, light production was normal (Fig. 4, curve 3 ).

\section{Correlation of prostaglandin synthesis and $C L$}

Aspirin inhibition of platelet $C L$. The influence of aspirin, a potent inhibitor of prostaglandin synthetase, on platelet CL, was studied by incubating C-PRP with $100 \mu \mathrm{M}$ of aspirin at $22^{\circ} \mathrm{C}$ for $20 \mathrm{~min}$. The platelets were separated from the plasma as usual and CL reaction initiated with either arachidonic acid or linoleic acid at 0 time. Fig. 5A shows that aspirin markedly inhibited platelet CL initiated by either fatty acid. The inhibitory influence of aspirin on CL production could not be removed by twice washing the platelets. Because aspirin does not absorb light in the visible region, inhibition of CL by aspirin could not be explained by quenching of light.

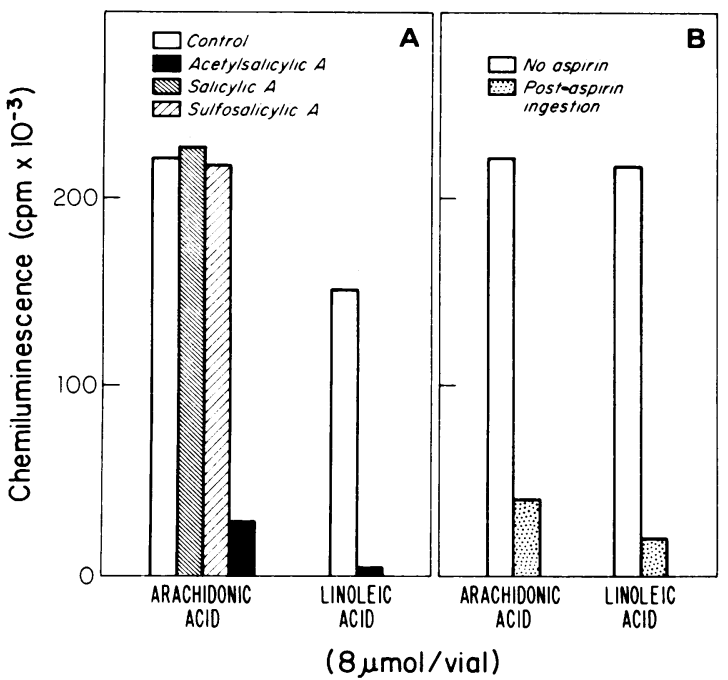

Figure 5 The effect of salicylates on platelet CL induced by arachidonic acid or linoleic acid. All reaction mixtures were prepared and maintained under exactly the same conditions as in Fig. 1. (A) Homologous C-PRP was incubated with $100 \quad \mu \mathrm{Ml}$ of aspirin, salicylic or sulfosalicylic acid. (B) Same donor as in $\mathrm{A} 24 \mathrm{~h}$ after aspirin ingestion. Data from a second donor was entirely comparable.
To examine whether aspirin was inhibiting platelet CL through a nonspecific effect rather than through the specific acetylation of cyclooxygenase (18), two compounds of similar structure to aspirin, but lacking cyclooxygenase effect, were chosen as controls. When platelets were incubated with salicylic acid or sulfosalicylic acid in the same concentrations and under the same conditions as aspirin-treated platelets, there was virtually no detectable inhibition of CL (Fig. 5A). These results would make a nonspecific effect of aspirin, such as metal chelation, highly unlikely. However, to confirm and extend the evidence that aspirin inhibition of CL was specific inhibition of cyclooxygenase, we studied the platelets of a donor who had ingested two 300 -mg tablets of acetylsalicylic acid $24 \mathrm{~h}$ previously. The effect of aspirin ingestion on platelet CL is shown in Fig. 5B. It is noteworthy that not only arachidonic acid- but linoleic acid-induced CL as well was markedly inhibited. Thus, it would appear that platelet CL induced by either the prostaglandin precursor, arachidonic acid, or by linoleic acid, which is not a prostaglandin precursor, involves the enzyme cyclooxygenase. As aspirin does not inhibit the conversion of arachidonic acid to 12-L-hydroxy-5,8,10,14-eicosatetraenoic acid via the lipoxygenase enzyme (19), this finding would exclude the lipoxygenase reaction (20) from participating in platelet CL.

Malondialdehyde production. When malondialdehyde production was used as an indication of platelet prostaglandin synthesis, a time-response curve of aspirin inhibition of arachidonic acid-induced platelet CL closely paralleled a time-response curve of aspirin inhibition of malondialdehyde production (Fig. 6). Similarly, the time-response curves of aspirin inhibition of platelet CL induced by linoleic acid closely paralleled that of arachidonic acid-induced platelet CL. There was incomplete inhibition by aspirin in both systems at the low concentration $(10 \mu \mathrm{M})$ of aspirin chosen. No detectable malondialdehyde was found on incubation of the linoleic acid with the platelets, a finding consistent with this fatty acid not being converted to a prostaglandin end product. These results clearly implicate cyclooxygenase as the enzyme involved in arachidonic-induced platelet CL. They further demonstrate that linoleic acid, a polyunsaturated fatty acid that is not a prostaglandin precursor, can induce electronically excited species capable of light emission and that this light production may involve activation of prostaglandin synthetase.

\section{DISCUSSION}

The present investigation has shown that isolated human platelets as well as platelet particulate fractions are capable of CL emission upon exposure to arachidonic acid. The light production is of rapid onset, peaking within 


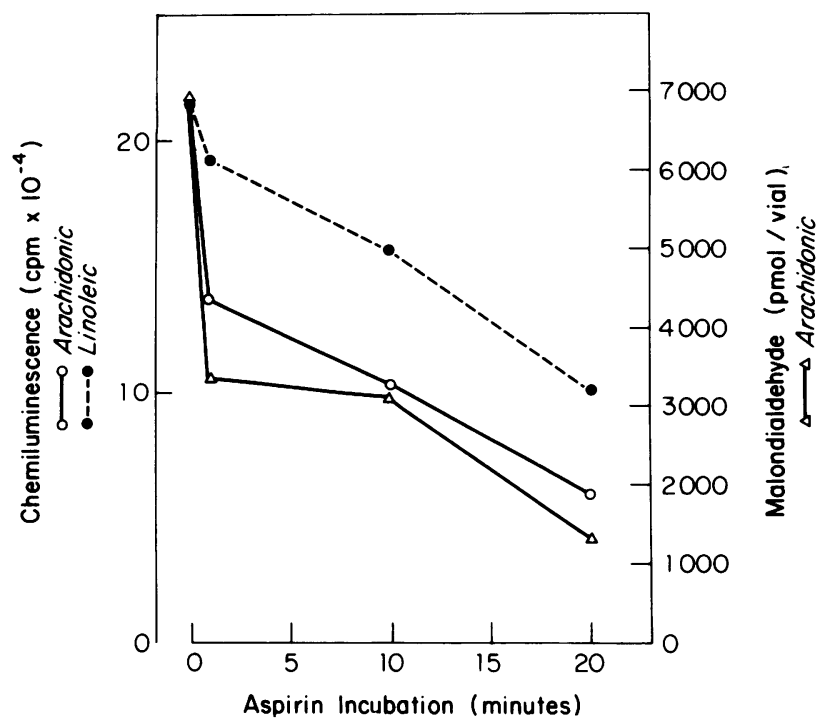

FIGURE 6 The effect of aspirin on platelet CL and malondialdehyde production. The reaction mixtures were prepared exactly as in Fig. 1 except that platelets were incubated with $10 \mu \mathrm{M}$ aspirin for the indicated length of time before the addition of arachidonic or linoleic acid. Incubation was carried out for 5 min during which time CL was measured. Immediately after incubation, the reaction mixtures were assayed for malondialdehyde production. Each point represents a duplicate experiment.

12-36 s after platelet-arachidonate incubation, and decaying to base line within 1-2 min. This time-course is similar to that reported for fatty acid-induced CL of sheep vesicular gland microsomes (9) and the NADPHdependent microsomal lipid peroxidation systems $(2$, 3 ), but occurs much more rapidly than human neutrophil CL $(5,7)$. The finding that the CL of the platelet system is almost entirely oxygen dependent is consistent with the findings in microsomal systems $(2,3)$ as well as for the CL of human leukocytes $(5,7)$ and rabbit macrophages (21).

Nakano and Sugioka $(2,3)$ showed by emission spectra that the luminescent species generated in their NADPH-dependent microsomal systems is ${ }^{1} \Delta \mathrm{g}$ type singlet oxygen as well as other electronically excited molecules in the triplet state, probably carbonyls. These products are thought to be generated by a selfreaction of lipid peroxy radicals. Allen (5) postulated that singlet oxygen is generated in phagocytizing leukocytes by two potential methods: the nonenzymatic disproportionation of $\mathrm{O}_{2}^{-}$and the reaction of the halidemyeloperoxidase-hydrogen peroxide system of the phagocyte. Allen proposed that the generated singlet oxygen, a potent electrophilic reactant, could attack electron-dense regions in unsaturated lipids, nucleic acid, and amino acid residues of the target microorganisms contained within the phagocytic vacuole. Oxidation of these substrates by singlet oxygen could gen- erate electronically excited carbonyl groups which relax by means of photon emission. Spectral analyses of phagocytosing granulocytes by both Cheson et al. (6) and Lint et al. (22) were unable to support the contention that singlet oxygen per se is the major emitter in this system because distinctive singlet oxygen emissions could not be detected. Rather, the essentially "white light" nature of the spectrum was consistent with oxidation of a variety of particle constituents producing a heterogenous group of electronically excited species. The investigators (6) emphasize that their study provides no information as to whether or not singlet oxygen is generated by phagocytosing granulocytes, and that it is not only possible but even likely that singlet oxygen is produced by these cells.

In the present study, the specific nature of the excited species responsible for platelet CL was not identified. Platelets have recently been shown to produce superoxide in small amounts (23). It was produced at a constant rate which did not increase after aggregation by agents such as collagen and thrombin. Platelet CL reported here was produced in a "burst" with light emission measurable in $>6 \mathrm{~s}$ after the reaction was initiated by arachidonate, peaking in $12-36 \mathrm{~s}$, and decaying to base line within 1-2 min. Platelet superoxide production was unaffected by prior ingestion of aspirin, indicating that the prostaglandin and thromboxane pathways are not involved. Platelet CL, on the other hand, was markedly inhibited by aspirin, and aspirin inhibition of the luminescence closely paralleled the time-course of aspirin inhibition of malondialdehyde production, findings which implicate the prostaglandin pathway. Thus, there is no evidence from these two studies to suggest that the superoxide radical is involved in the generation of platelet CL. On the other hand, the present study provides no information as to whether or not superoxide is involved in platelet CL emission.

The present study has evaluated the relationship between arachidonic acid-induced platelet CL and platelet prostaglandin biosynthesis. Evidence that these two events are related comes from several findings. Both events are found in the same subcellular fraction. In the present study, a significant proportion of platelet CL was localized in platelet particulate fraction. Previously, we have reported (24) that prostaglandin synthesis occurs in this subcellular fraction. Further evidence that platelet CL and prostaglandin synthesis are related is the finding that both are strictly oxygen dependent. This oxygen dependency, plus the very early "burst" in light emission, would be consistent with a light-emitting species participating in the oxidation reaction of arachidonate, early in prostaglandin synthesis.

The most compelling evidence that platelet CL is related to the prostaglandin pathway and specifically involves the activation of cyclooxygenase comes from 
studies employing aspirin inhibition (Figs. 5 and 6). Low concentrations of acetylsalicylic acid have been reported to acetylate prostaglandin synthetase specifically (18). We have shown that the addition of low concentrations of aspirin to platelet preparations markedly inhibits arachidonate-induced CL. This inhibition cannot be removed by washing and cannot be explained on the basis of light quenching. Further, platelets from a donor who ingested aspirin $24 \mathrm{~h}$ previously had markedly reduced CL production in this system. Inasmuch as the inhibitory effect of aspirin is specifically acetylation of prostaglandin synthetase, structurally similar compounds such as salicylic acid or sulfosalicylic acid, which lack an acetyl group, would not be expected to inhibit this enzyme. These compounds had absolutely no influence on platelet CL. In addition, we found that a time-response curve of aspirin inhibition of arachidonate-induced CL closely paralleled a time-response curve of aspirin inhibition of malondialdehyde production (Fig. 6). These studies of aspirin inhibition of platelet CL, and the temporal relationship of this inhibition with aspirin inhibition of malondialdehyde formation, strongly implicate cyclooxygenase activation in the CL production. They suggest that electronically excited species may be involved in the initial oxidation of arachidonate or in its conversion to the endoperoxides, prostaglandin $\mathrm{G}_{2}$ and prostaglandin $\mathrm{H}_{2}$. Inhibition of platelet CL by aspirin (in concentrations adequate to completly inhibit the cyclooxygenase) dissociates the platelet CL from the platelet lipoxygenase reaction (20) because aspirin does not inhibit the conversion of 12 -L-hydroxy-5,8,10,14 eicosatetraenoic acid via the lipoxygenase enzyme (19). However, the small proportion of CL not inhibited by aspirin, or by boiling the platelets, is not explained and appears due to a reaction separate from prostaglandin synthesis.

In addition to arachidonic acid, eight other polyunsaturated fatty acids, only three of which are known to be prostaglandin precursors, induced platelet CL. Evidence that these fatty acids, particularly linoleic acid, which are not prostaglandin substrates, may specifically interact with prostaglandin synthetase comes from the studies of aspirin inhibition of linoleic acidinduced platelet CL. The addition of low concentrations of aspirin to platelet preparations markedly inhibited linoleic acid-induced CL (Fig. 5) in much the same manner as the inhibition noted for arachidonateinduced CL. Similarly, platelets from a donor who ingested aspirin $24 \mathrm{~h}$ previously had markedly reduced CL production in this system. Moreover, the timecourse of aspirin inhibition of linoleic acid-stimulated CL paralleled the aspirin inhibition of arachidonateinduced CL. These findings suggest that either linoleic acid undergoes a specific interaction with prostaglandin synthetase with the resultant conversion to an elec- tronically excited species but without achieving a final conversion to prostaglandin, or it interacts with a separate platelet enzyme (inhibitable by aspirin) to produce CL. This finding may have implications for the mechanism by which linoleic acid inhibits the conversion of arachidonic acid to prostaglandin endoperoxides and thromboxane $\mathrm{A}_{2}$ (25).

\section{ACKNOWLEDGMENTS}

The authors are grateful to Ken Rholl and Steve Stoddard for their excellent technical assistance and to Patti Lorenz for secretarial support.

This work was supported in part by U. S. Public Health Service grants AI 08821, AI 06931, HL 11880, CA 11996, CA 21737, and CA 07376.

\section{REFERENCES}

1. Howes, R. M., and R. H. Steele. 1972. Microsomal $(\mu \mathrm{S})$ chemiluminescence (CL) induced by NADPH and its relation to aryl-hydroxylations. Res. Commun. Chem. Pathol. Pharmacol. 3: 349-357.

2. Nakano, M., T. Noguchi, K. Sugioka, H. Fukyama, M. Sato, Y. Shimizu, Y. Tsuji, and H. Inaba. 1975. Spectroscopic evidence for the generation of singlet oxygen in the reduced nicotinamide adenine dinucleotide phosphate-dependent microsomal lipid peroxidation system. J. Biol. Chem. 250: 2404-2406.

3. Sugioka, K., and M. Nakano. 1976. A possible mechanism of the generation of singlet molecular oxygen in NADPHdependent microsomal lipid peroxidation. Biochim. Biophys. Acta. 423: 203-216.

4. Goda, K., J. W. Chu, T. Kimura, and P. A. Schaap. 1973. Cytochrome c enhancement of singlet molecular oxygen production by the NADPH-dependent adrenodoxin reductase-adrenodoxin system: the role of singlet oxygen in damaging adrenal mitochondrial membranes. Biochem. Biophys. Res. Commun. 52: 1300-1306.

5. Allen, R. C., R. L. Stjernholm, and R. H. Steele. 1972. Evidence for the generation of an electronic excitation state(s) in human polymorphonuclear leukocytes and its participation in bactericidal activity. Biochem. Biophys. Res. Commun. 47: 679-684.

6. Cheson, B. D., R. L. Christensen, R. Sperling, B. E. Kohler, and B. M. Babior. 1976. The origin of chemiluminescence of phagocytosing granulocytes. J. Clin. Invest. 58: 789-796.

7. Nelson, R. D., E. L. Mills, R. L. Simmons, and P. G. Quie. 1976. Chemiluminescence response of phagocytizing human monocytes. Infect. Immun. 14: 129-134.

8. Sagone, A. L., Jr., G. W. King, and E. N. Metz. 1976. A comparison of the metabolic response of phagocytosis in human granulocytes and monocytes. J. Clin. Invest. 57: $1352-1358$.

9. Marnett, L. J., P. Wlodawer, and B. Samuelsson. 1974. Light emission during the action of prostaglandin synthetase. Biochem. Biophys. Res. Commun. 60: 12861294.

10. Horns, D. J., J. M. Gerrard, G. H. R. Rao, W. Krivit, and J. G. White. 1976. Smoking and platelet labile aggregation stimulating substance (LASS) synthesizing activity. Thromb. Res. 9: 661-668.

11. Stanley, D. E., and S. G. Williams. 1969. Use of the scintillation spectrometer for determining adenosine triphosphate by the luciferase enzyme. Anal. Biochem. 29: $381-392$. 
12. Stjernholm, R. L., R. C. Allen, R. H. Steele, W. W Waring, and J. A. Harris. 1973. Impaired chemiluminescence during phagocytosis of opsonized bacteria. Infect. Immun. 7: 313-314.

13. Smith, J. B., C. M. Ingerman, and M. J. Silver. 1976 Malondialdehyde formation as an indicator of prostaglandin production by human platelets. J. Lab. Clin. Med. 86: 167-172.

14. Dejesus, M., Jr., S. Fikrig, and T. Detwiler. 1972. Phagocytosis-stimulated nitroblue tetrazolium reduction by platelets. J. Lab. Clin. Med. 80: 117-124.

15. Bergstrom, S., H. Danielsson, and B. Samuelsson. 1964 The enzymatic formation of prostaglandin $E_{2}$ from arachidonic acid. Biochim. Biophys. Acta. 90: 207-210.

16. Tobias, L. D., F. M. Vane, and J. R. Paulsrud. 1975. The biosynthesis of $1_{a}, l_{b}$-D1HOMO-PGE $E_{2}$ and $l_{a}, l_{b}$ DIHOMO-PGF ${ }_{2} \alpha$ from 7, 10, 13, 16-docosatetranaenoic acid by an acetone-pentane power of sheep vesicular gland microsomes. Prostaglandins. 10: 443-468.

17. Levine, P. H., K. L. Scoon, J. C. Hardin, and N. I. Krinsky. 1977. The source of hydrogen peroxide and of chemiluminescence observed in activated human platelet preparations. Thrombosis and Haemostasis Abstract. 6th International Congress on Thrombosis and Haemostasis. 210.

18. Roth, G. J., and P. W. Majerus. 1975. The mechanism of the effect of aspirin on human platelets. I. Acetyla- tion of a particulate fraction protein. J. Clin. Invest. 56 $624-632$.

19. Hamberg, M., and B. Samuelsson. 1974. Prostaglandin endoperoxides: novel transformations of arachidonic acid in human platelets. Proc. Natl. Acad. Sci. U. S. A 71: 3400-3404.

20. Nugteren, D. H. 1975. Arachidonate lipoxygenase in blood platelets. Biochim. Biophys. Acta. 380: 299-307.

21. Allen, R. C., and L. D. Loose. 1976. Phagocytic activation of a luminol-dependent chemiluminescence in rabbit alveolar and peritoneal macrophages. Biochem. Biophys. Res. Commun. 69: 245-252.

22. Lint, T. F., A. M. Brendzel, and B. R. Anderson. 1977. Studies on the spectral characteristics of the chemiluminescent emissions from human polymorphonuclear leukocytes and isolated myeloperoxidases. Fed. Proc. 36: 1071 .

23. Marcus, A. J., S. T. Silk, L. B. Safier, and H. L. Ullman. 1977. Superoxide production and reducing activity in human platelets. J. Clin. Invest. 59: 149-158.

24. Gerrard, J. M., J. G. White, G. H. R. Rao, and D. Townsend. 1976. Localization of platelet prostaglandin production in the platelet dense tubular system. Am.J. Pathol. 83: 283-298.

25. Gerrard, J. M., J. G. White, and W. Krivit. 1976. Labile aggregation stimulating substance, free fatty acids, and platelet aggregation. J. Lab. Clin. Med. 87: 73-82. 